\title{
Irregular Wave Modelling with CFD in Sulafjord for the E39 Project
}

\author{
Weizhi Wang, Norwegian University of Science and Technology, weizhi.wang@ntnu.no \\ Arun Kamath, Norwegian University of Science and Technology, arun.kamath@ntnu.no \\ Hans Bihs, Norwegian University of Science and Technology, hans.bihs@ntnu.no
}

\begin{abstract}
INTRODUCTION
The E39 project aims at building a continuous ferry-free coastal highway along the west coast of Norway. Sulafjord is one of the fjords where ferries are to be replaced with floating bridges or floating tunnels. The floating structures demand accurate and realistic numerical simulations of the wave propagation and transformation in the fjords. The Norwegian coastline is characterized by dramatic water depth changes and deep water conditions. The coastal water also contains both swells and local wind-generated waves. These conditions, along with series of islands outside the fjords and very irregular coastline, make wave modeling more challenging for this region. Thus, the application of CFD models which provide high-resolution and phase-resolved solutions for complicated wave freesurface is explored. First, the spectral wave model SWAN is used to estimate the wave properties at the inlet of the Sulafjord from offshore wave data. Using the estimated wave data at the inlet as input, a large-scale 3D regular wave CFD simulation is performed using the open-source model REEF3D. Then unidirectional and multi-directional irregular wave CFD simulations are performed to represent a more realistic sea state, using a frequency spectra and a directional spreading function. The statistical properties of the simulated irregular ocean waves at three locations inside the fjord are compared among the CFD simulations and with the spectral wave model. The differences in the simulation results are discussed and studied.
\end{abstract}

\section{NUMERICAL MODEL}

The incompressible Navier-Stokes equations are solved with the CFD model REEF3D::CFD to describe the flow properties in the domain. REEF3D applies a fifth-order WENO scheme for spatial discretization of the convection terms and a third-order TVD Runge-Kutta scheme for the temporal terms. The level-set method is used to capture the free surface. The local inverse distance interpolation method is applied for obtaining the bottom topography. A relaxation method is adopted for the wave generation and absorption. The code is parallelized using MPI.

\section{METHODOLOGY}

First, a simulation over the entire continental shelf area off Sulafjord is conducted with the spectral wave model SWAN, using the offshore long-term wave data as input. The wave properties at the entrance of the fjord are obtained from the spectral wave model simulation and are used as input wave data in the CFD numerical tanks. A regular wave, a unidirectional irregular wave and a multidirectional irregular wave are generated in a numerical wave tank with REEF3D over the irregular bottom topography at Sulafjord. A JONSWAP power spectrum is used to generate the unidirectional irregular wave. Combining the JONSWAP spectrum and the frequencyindependent Mituyasu directional spreading function, a multi-directional irregular wave is also generated. A double summation method is used to consider all combinations of frequency components and directional components in the multi-directional irregular wave generation. The significant wave heights $H_{s}$ at three locations inside the Sulafjord are also compared among the different CFD simulations and with the spectral wave model simulation. The probe locations and the computational domain are shown in Fig. 1. All CFD simulations are run for 1800 seconds, and the significant wave heights $H_{s}$ are calculated using the last 1000 seconds' time series for all three probes.

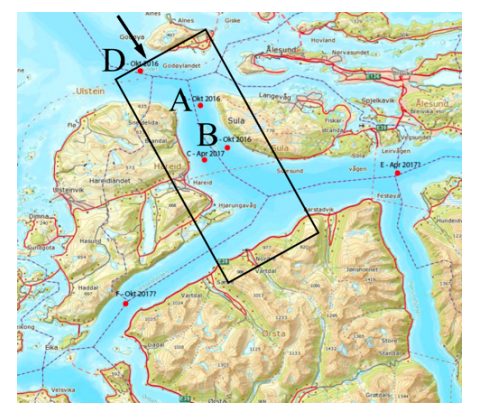

Figure 1 - The map of Sulafjord and the defined wave probes and computational domain. The black box is the computational domain for the CFD simulations. The three probes are located at $D, A$ and $B$, where the significant wave heights $H_{s}$ are compared. The arrow indicates the incoming wave direction.

\section{RESULTS AND DISCUSSION}

The significant wave height distribution from the spectral wave model is shown in Fig. 2. The detailed wave freesurface is not captured, but the transport of wave energy is well illustrated. The regular wave, unidirectional irregular wave and multi-directional irregular wave CFD simulation results are shown in Fig. 3 to Fig. 5. As can be seen from the figures, the wave refraction, diffraction and reflection because of the irregular bottom and the irregular coastline are clearly demonstrated in the phase-resolved model. The significant wave heights from the different simulations are compared in Table 1. In the irregular wave simulations, the significant wave heights are calculated based on the spectral information at the probes. In the regular wave simulation, the time series of wave height appears very irregular due to the complicated wave transformation. Therefore, the average wave height of the highest $1 / 3$ waves is calculated for the comparison. It is observed that in the unidirectional wave field, the wave height in the center of the fjord is higher, while the wave height at the side of the fjords is lower. The wave heights in the multidirectional wave field appear to be more evenly distributed inside the fjord. Because of the complex wave patterns, the $H_{S}$ tend to be higher at some locations due to standing wave pattern or wave focusing, and lower at other locations due to the wave-wave interaction and the cancellation of the waves. For example, in the 
unidirectional irregular wave simulation, the $H_{S}$ is lower than input wave at probe $\mathrm{D}$, while in the multi-directional irregular wave simulation, the $H_{s}$ tends to decrease at location $\mathrm{B}$. This complicated variation of $H_{S}$ due to the combined effect of refraction, diffraction and reflection is well captured by the high-resolution phase-resolved model REEF3D. The spectral wave simulation gives very low wave height inside the fjord because the spectral wave model is not able to treat diffraction accurately and the wave energy is not well represented after the waves enter the fjord. Different wave types have distinguishing wave behavior inside the fjord. For local wind-generated waves, the multi-directional irregular wave modelling can better represent the reality.

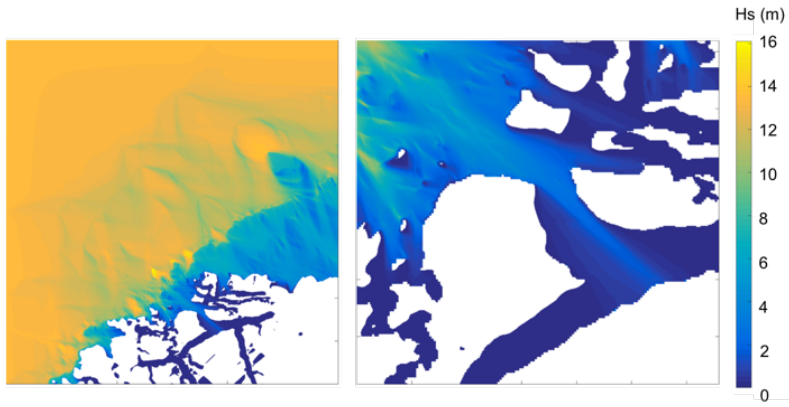

Figure 2 - The distribution of significant wave height in the SWAN simulation in Sulafjord. The overview of the large continental shelf domain is shown on the left and the focused view at the fjord is shown on the right

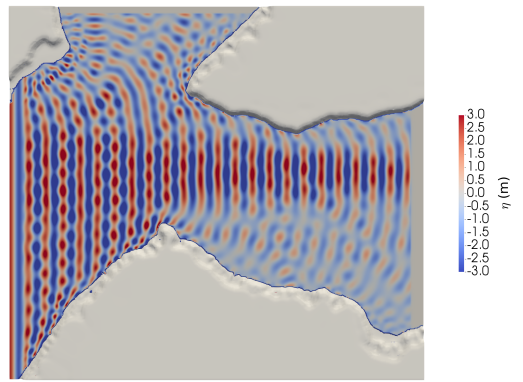

Figure 3 - Free-surface elevation of the regular wave CFD simulations in Sulafjord at $\mathrm{t}=1800 \mathrm{~s}$

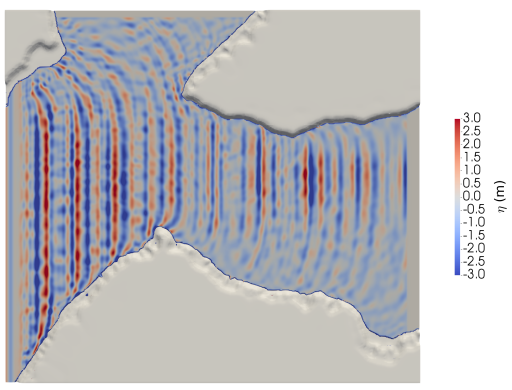

Figure 4 - Free-surface elevation of the unidirectional irregular wave CFD simulation in Sulafjord at $t=1800 \mathrm{~s}$

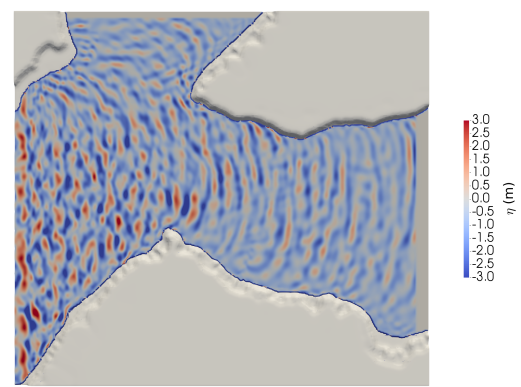

Figure 5 - Free-surface elevation of the multi-directional irregular wave CFD simulation in Sulafjord at $t=1800 \mathrm{~s}$

Table 1 - Comparison of wave heights (m) using different numerical models

\begin{tabular}{c|c|c|c|c}
\hline Probes & SWAN & $\begin{array}{c}\text { Regular } \\
\text { wave } \\
\text { CFD }\end{array}$ & $\begin{array}{c}\text { Uni- } \\
\text { directional } \\
\text { Irregular } \\
\text { wave CFD }\end{array}$ & $\begin{array}{c}\text { Multi- } \\
\text { directional } \\
\text { irregular } \\
\text { wave CFD }\end{array}$ \\
\hline D & 4.67 & 5.2364 & 4.6961 & 5.3888 \\
\hline A & 2.57 & 5.0318 & 5.6303 & 5.4232 \\
\hline B & 2.28 & 5.3650 & 5.5274 & 4.4725 \\
\hline
\end{tabular}

\section{CONCLUSIONS}

3D large-scale wave modelling in Sulafjord using the opensource CFD model REEF3D is presented. Different waves are generated and propagate over an irregular bottom topography in the numerical wave tank. The wave transformation due to refraction, diffraction and reflection phenomena are well represented in the CFD simulations. The diffraction and reflection of the ocean waves insert more complexity in the wave field and may cause particular distributions of $H_{S}$. With directional spreading, the wave patterns are closer to the natural situation and local windgenerated waves can be better represented. Compared to the phase-resolved CFD simulation, spectral model proves to be ideal for large-scale simulations with simple topography and boundary conditions but fails at locations with strong diffraction and reflection. The study demonstrates the capability of the wave model REEF3D in simulating 3D irregular wave field over irregular bottom. The result shows the significance of applying phaseresolved models in the irregular coastal zone. It also addresses the significance of multi-directional irregular wave simulation for a more realistic representation of coastal waves, especially of wind-generated waves. Further studies to improve the accuracy of the phaseresolved multi-directional irregular wave simulation will be conducted in the future.

\section{ACKNOWLEDGEMENTS}

This study has been carried out under the E39 fjord crossing project (No. 304624) and the authors are grateful to the grants provided by the Norwegian Public Roads Administration and the computational resources provided by NOTUR (NN2620K) at NTNU. 